\title{
Preparation and Characterization of Chitosan-Humic Acid-Zerovalent Iron Nanocomposite for Nitrate Reduction in Water
}

\author{
Caroline Avosuahi Akinremi, ${ }^{1}$ Nikechukwu Nike Omosun, ${ }^{2}$ Sheriff Adewuyi, ${ }^{1}$ \\ Jamiu Oladipupo Azeez, ${ }^{3}$ and Sanyaolu Nurudeen Olanrewaju ${ }^{1}$ \\ ${ }^{1}$ Department of Chemistry, Federal University of Agriculture Abeokuta, PMB 2240, Abeokuta, Ogun State 110001, Nigeria \\ ${ }^{2}$ Department of Chemistry, Federal University of Agriculture, PMB 7257, Umudike, Abia State 440109, Nigeria \\ ${ }^{3}$ Soil Science and Land Management, Federal University of Agriculture Abeokuta, PMB 2240, Abeokuta, Ogun State 110001, Nigeria
}

Correspondence should be addressed to Caroline Avosuahi Akinremi; cakinremi2002@yahoo.com

Received 20 April 2016; Revised 25 July 2016; Accepted 14 August 2016

Academic Editor: Khalid Z. Elwakeel

Copyright (C) 2016 Caroline Avosuahi Akinremi et al. This is an open access article distributed under the Creative Commons Attribution License, which permits unrestricted use, distribution, and reproduction in any medium, provided the original work is properly cited.

\begin{abstract}
A new zerovalent iron chitosan-humic acid nanocomposite was prepared and tested for nitrate ion reduction in water. Humic acid was used for intramolecular cross-linking of the chitosan linear chains to increase the active sites on the chitosan biopolymer and then further used as a stabilizer to synthesize zerovalent iron nanoparticles by the reduction of iron (II) chloride with sodium borohydride. Characterization of the products was carried out using infrared spectroscopy, scanning electron microscope, energy dispersive X-ray, and X-ray diffractometer. Batch experiments were conducted for the reduction of nitrate in water using different concentrations of the products in different concentrations of nitrate ion and at different contact time. The adsorption equilibrium data for the nitrate solution gave a favorable adsorption according to the Langmuir equation. Varying the nanocomposite-to-nitrate ion ratio generally led to faster nitrate reduction, with the pseudofirst-order rate constant for the adsorption increasing with increase in nanocomposite-to-nitrate ion ratio. Nitrate removal efficiency of zerovalent iron chitosan-humic acid nanocomposite was further confirmed using real water samples obtained from drainage waste and river with an initial nitrate concentration of $18.00 \pm 0.01$ and $12.00 \pm 0.12 \mathrm{ppm}$, respectively. The reduction of nitrate in water using the nanocomposite was concluded to be highly effective.
\end{abstract}

\section{Introduction}

There has been an increase in the discharge of toxic substances such as nitrate into the environment especially water bodies as a result of urbanization. Nitrate anion pollution has been attributed to agricultural runoff, leaching of nitrogen fertilizers, concentrated animal feeding operations, food processing, and industrial waste effluent discharge $[1,2]$. In the area of water purification, nanotechnology offers the possibility of an efficient removal of pollutants and germs. The application of zerovalent metal nanoparticles (Nps) for environmental remediation represents one of the innovative technologies especially in the chemical reduction of some oxoanions from drinking water and destruction of some organic pollutants [3-5]. Nanoscale bimetallic particles have been shown to remove some pollutants [6].
Kumar and Chakraborty [7] have proposed that the reduction of nitrate by some zerovalent nanoparticles gives rise to ammonia or nitrogen, water, or hydroxyl ion. This could occur in the presence of protons. Biopolymers have been used to stabilize the zerovalent metals Nps which resulted in more effective nanocomposites, examples of which are watersoluble starch [8], chitosan, and its derivatives [2,9]. Chitosan (CTS), as one of the most abundant natural polymers, is nontoxic, biodegradable and biocompatible. Different from most other natural polymers, chitosan has high reactivity and processability for its specific molecular structure and polycationic nature. Humic acid (HMA) is a principal component of humic substances. Humic substances have been studied for their possible application in remediation of polluted environments $[10,11]$. HMA has been reported to 
have enormous chelation capacity due to the presence of hydroxyl-, phenoxyl-, and carboxyl-reactive groups which predominantly carry negative charges $[12,13]$ making it possible for further reactions with other agents. This work hitherto describes the synthesis of zerovalent iron crosslinked chitosan-humic acid nanocomposite and its effective nitrate ion reduction in waste water.

\section{Materials and Method}

2.1. Materials. Chitosan (average molecular weight of $4294.40 \mathrm{~g} / \mathrm{mol}$ and $80 \%$ degree of deacetylation) was prepared from chitin which was obtained from snail shells as previously reported [2]. Sodium borohydride $\left(\mathrm{NaBH}_{4}\right)$, humic acid, and N-3-(dimethylaminopropyl)- $\mathrm{N}^{1}$-1-ethylcarbodiimide (CDI) were purchased from Sigma Aldrich, United State. Other reagents like ferrous chloride hydrate $\left(\mathrm{FeCl}_{2} \cdot 4 \mathrm{H}_{2} \mathrm{O}\right), \mathrm{NaOH}, \mathrm{HCl}$, acetone, acetic acid, and $\mathrm{NaNO}_{3}$ were obtained from $\mathrm{BDH}$. All aqueous solutions were made with ultrahigh purity water purified with an ultrapure water system Milli-Q Plus (Millipore Co.).

2.2. Characterization. The degree of deacetylation (DDA) of CTS was determined using the acid-base titration method [14]. The IR spectra were recorded on a Perkin-Elmer 100 FT-IR spectrophotometer. The XRD patterns of CTS and CTS-HMA were obtained on Philips PWO4XPert pro X-ray diffractometer. The X-ray source was $\mathrm{Cu} \mathrm{K} \alpha$ with a voltage of $40 \mathrm{kV}$ and a current of $30 \mathrm{~mA}$. The morphology of the products was studied by the Tescan (TS5136ML) scanning electron microscope (SEM) operating at an accelerated voltage of $20 \mathrm{kV}$ after a gold sputter coating at $10 \mu \mathrm{m}$ and the elements present in the nanocomposites determined by energy dispersive X-ray (EDX) element mapping. UV-spectrophotometer used was Spectrumlab $752 \mathrm{~S}$ UV-Vis spectrophotometer.

\subsection{Synthesis of CTS-HMA and ZvFe-CTS-HMA Nanocom-} posite. Following a previously described procedure [9, 15] $8 \mathrm{~g}$ CTS was dissolved in HMA solution and stirred with a magnetic stirrer for $2 \mathrm{~h}$. Few drops of water-soluble carbodiimide were added to the solution and further stirred for $2 \mathrm{~h}$ at room temperature and were stored at room temperature for $24 \mathrm{~h}$. The cross-linked CTS-HMA was obtained by freeze drying. The synthesis of ZvFe-CTS-HMA nanocomposite is based on borohydride reduction of $\mathrm{Fe}^{2+}$. To a stirred ethanolic solution of $\mathrm{FeCl}_{2} \cdot 4 \mathrm{H}_{2} \mathrm{O}$ (10.68 g) CTS-HMA (3.0 g) was added. $1 \mathrm{M} \mathrm{NaBH}_{4}$ solution was then added drop-wise to the Fe-CTS-HMA mixture with continuous stirring of the resulting solution (Scheme 1). The nanocomposite was collected by freeze drying.

2.4. Reduction of Nitrate in Water. Kinetic studies were carried out to evaluate the efficiency of reduction of nitrate by the products on both standards and samples collected from river and waste water. All experiments were carried out in duplicate. Control experiments were also carried out in parallel.
2.4.1. Batch Experiment. Batch tests for nitrate reduction were conducted in a $20 \mathrm{~mL}$ sterilized, disposable centrifuge tubes. Different concentrations of nitrate were prepared from a stock solution of $1000 \mathrm{ppm}$ ranging from $10 \mathrm{ppm}$ to $50 \mathrm{ppm}$ using double distilled and deionized water. CTS, HMA, CTSHMA, and ZvFe-CTS-HMA of $0.1 \mathrm{~g}$ dosage were added to $20 \mathrm{~mL}$ each of varying concentrations of nitrate at different selected time intervals of 15, 30, 45, 60, 75, and 90 minutes; the mixtures were shaken and centrifuged at $400 \mathrm{rpm}$. At selected time intervals, $2 \mathrm{~mL}$ samples were taken using a gas tight syringe and nitrate concentration was determined using $\mathrm{UV}$-visible spectrophotometer [16]. An increase in dosage to $0.2 \mathrm{~g}$ and $0.4 \mathrm{~g}$ was also carried out using high concentration of nitrate of $50 \mathrm{ppm}$ at different time intervals.

2.4.2. Nitrate Reduction in River and Waste Water Samples. Samples were collected randomly in polyethylene bottles. Nitrate concentration was determined before and after the reduction using the synthesized zerovalent iron cross-linked chitosan at $0.1 \mathrm{~g}$ and $0.2 \mathrm{~g}$ dosages as carried out in the batch experiments.

2.4.3. Reaction Kinetics and Adsorption Isotherms. A pseudofirst-order reaction with respect to nitrate concentration was used to fit the kinetic data as given in (1), where $k$ is the observed first-order reaction rate constant, which is the slope of the regression lines by plotting a natural log graph with respect to nitrate concentration and reaction time $\left(\ln \left[\mathrm{NO}_{3}{ }^{-}\right]\right.$ versus time) according to (2). A pseudosecond-order reaction with respect to nitrate concentration was also used to fit the observed kinetic data as given in (3). Thus, plot of $\ln \left[\mathrm{NO}_{3}{ }^{-}\right]$ versus time should yield a straight line, and the slope $k$ is the pseudosecond rate constant.

$$
\begin{aligned}
r & =\frac{-d[A]}{d t}=k[A], \\
\ln [A] & =-k_{1} t[A]_{0}, \\
r & =\frac{-d[A]}{d t}=k_{2}[A]^{2}, \\
\frac{1}{[A]} & =\frac{1}{[A]_{0}}+k t .
\end{aligned}
$$

To examine the relationship between sorbed $(q)$ and aqueous concentrations $\left(C_{e}\right)$ at equilibrium, sorption isotherm models are employed. Freundlich equation was tested by plotting $\log q_{e}$ against $\log C_{e}$; according to (3), such a plot should yield a straight line with intercept equal to $\log K_{f}$ and slope equal to $1 / n$. The Langmuir isotherm was also tested by plotting $C_{e} / q_{e}$ against $C_{e}$ according to (5). The applicability of the isotherm to the adsorption was judged by comparing the values of regression coefficient $\left(R^{2}\right)$.

$$
\begin{aligned}
& \frac{C_{e}}{q_{e}}=\frac{1+C_{e}}{q_{0} k_{L} q_{0}}, \\
& R_{L}=\frac{1}{1+b C_{0}} .
\end{aligned}
$$




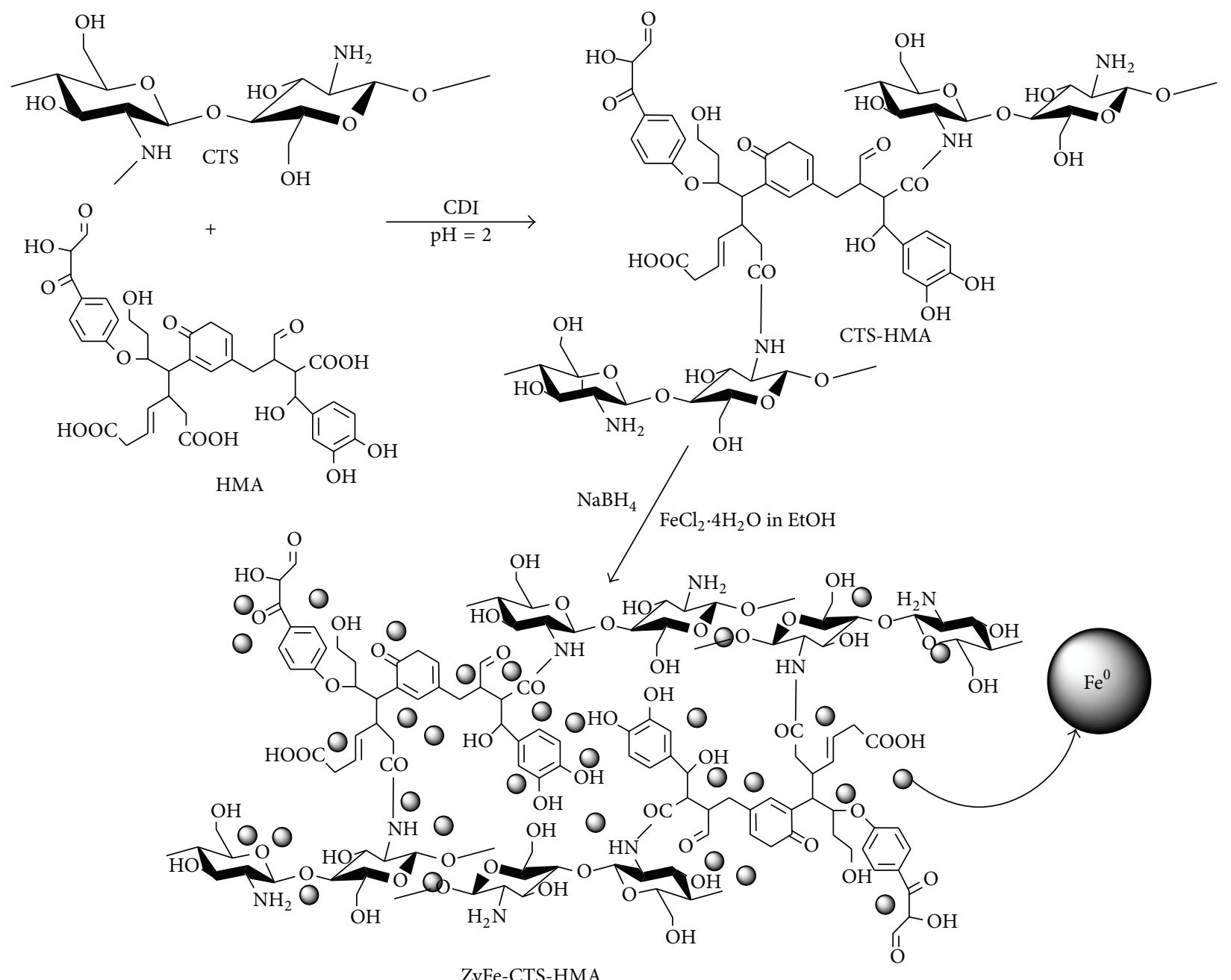

Scheme 1: Proposed synthetic pathway of preparation of ZvFe-CTS-HMA.

The equilibrium parameter, $R_{L}$, of the Langmuir isotherm was calculated (6) as has been previously defined [17], where $\mathrm{C}_{0}$ in the initial $\mathrm{NO}_{3}{ }^{-}$concentration (ppm) and $b$ is Langmuir adsorption equilibrium constant $\left(\mathrm{mL} \mathrm{mg}^{-1}\right)$.

\section{Results and Discussions}

3.1. Synthesis and Characterization. The zerovalent iron chitosan-humic acid nanocomposite was precipitated out as a powder and therefore could be dispersed into aqueous solutions. The synthesized ZvFe-CTS-HMA was stable when exposed to open atmosphere for long period of hours. It is believed that, in the humic acid-chitosan system, the zerovalent iron nanoparticles are better protected by the CTSHMA and oxidation prevented as previously reported with other cross-linked chitosans [9].

IR spectrum of chitosan obtained in this study was comparable to those previously reported $[2,9,18]$. Figure 1 compares the infrared assignment to functional groups of CTS, HMA, CTS-HMA, and ZvFe-CTS-HMA. The broad peak around $3448-3373 \mathrm{~cm}^{-1}$ in the spectrum of CTS, HMA, and CTS-HMA samples indicated the absence of free $\mathrm{OH}$ groups [19]. A new weak peak at $1654 \mathrm{~cm}^{-1}$ in chitosan-humic acid was assigned to be characteristic $-\mathrm{COO}^{-}$symmetric stretching vibration of carboxylate anion. This is comparable to that observed by Santosa et al. [18], where chitosan was cross-linked with glutaraldehyde. It was suggested that the amine groups of chitosan reacted with the carboxylic groups of humic acid electrostatically, this is because carboxylic groups of humic acid release protons $\left(\mathrm{H}^{+}\right)$and convert to $\mathrm{COO}^{-}$anions, whereas amine groups of chitosan as a base can accept protons and convert to cations. Hence, crosslinking between humic acid and chitosan can be considered to occur chemically. Comparing the IR spectra of CTS-HMA and $\mathrm{ZvFe}-\mathrm{CTS}-\mathrm{HMA}$, it is evident that there is a slight band shift of absorption peaks of carbonyl stretching occurring at $1831 \mathrm{~cm}^{-1}$ and the amide I and amide II bands occurring at 1475 and $1391 \mathrm{~cm}^{-1}$, respectively. A weak peak of free $-\mathrm{OH}$ appeared, which is attributed to electrostatic interaction of $\mathrm{Fe}$ with $\mathrm{OH}$ and $\mathrm{NH}_{2}$ group of CTS-HMA. The observed shift in 


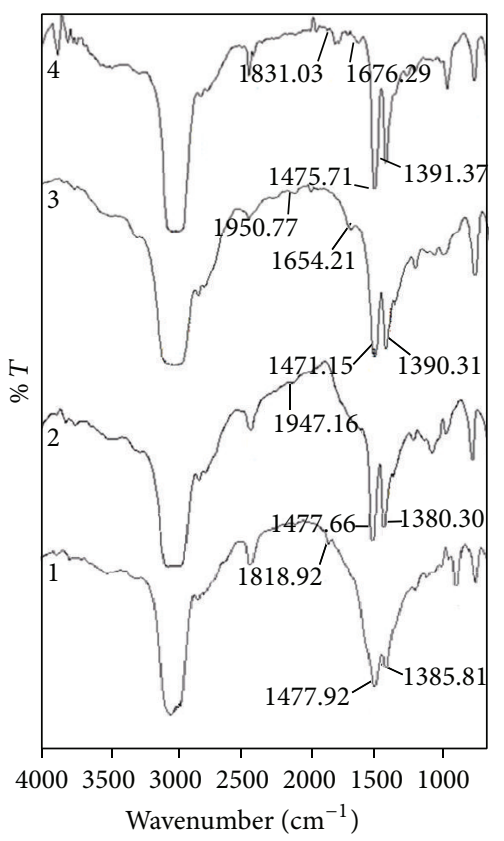

FIgURE 1: Infrared spectra: 1: CTS; 2: HMA; 3: CTS-HMA; 4: ZvFeCTS-HMA.

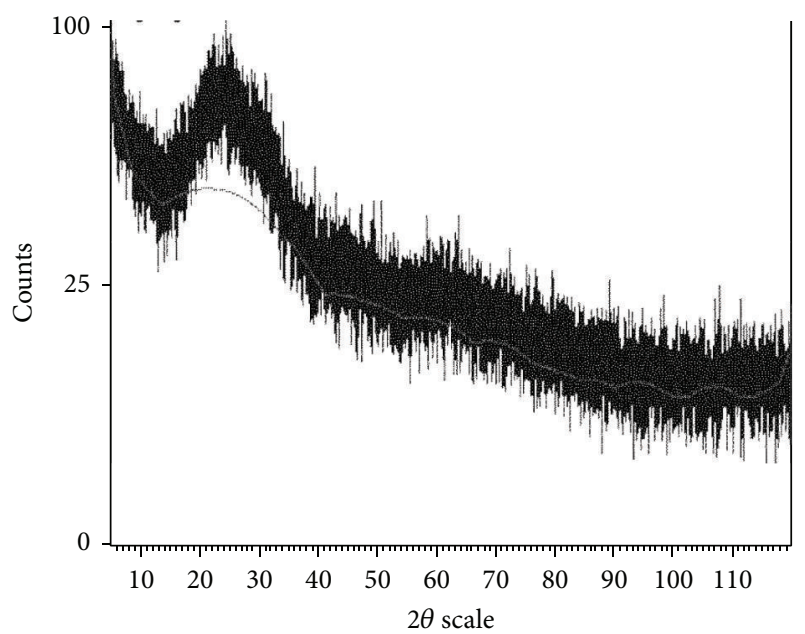

Figure 2: X-ray diffractogram of ZvFe-CTS-HMA.

the position of the absorption bands and the appearance of two novel bands in the IR spectra of CTS-HMA and ZvFeCTS-HMA showed that ZvFe-CTS-HMA was successfully synthesized.

The XRD data of ZvFe-CTS-HMA presented in Figure 2 shows a very broad peak and scattered features. The broad and scattered peak reveals the existence of an amorphous phase of the nanoparticle; therefore this cannot be defined by a crystalline model. The characteristic broad peak at $2 \theta$ of $25^{\circ}$ indicates that the zerovalent iron is predominantly present in the sample in an amorphous phase $[18,20]$. This is not surprising as humic acid is amorphous in nature and also the diffraction pattern of sorbent obtained by cross-linking between chitosan and humic acid showed an amorphous form.

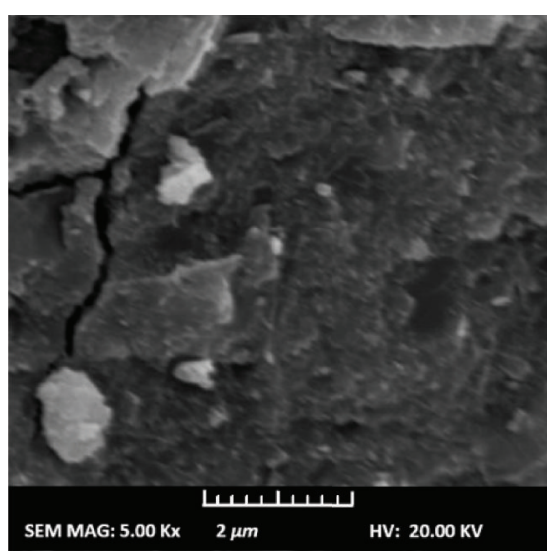

(a)

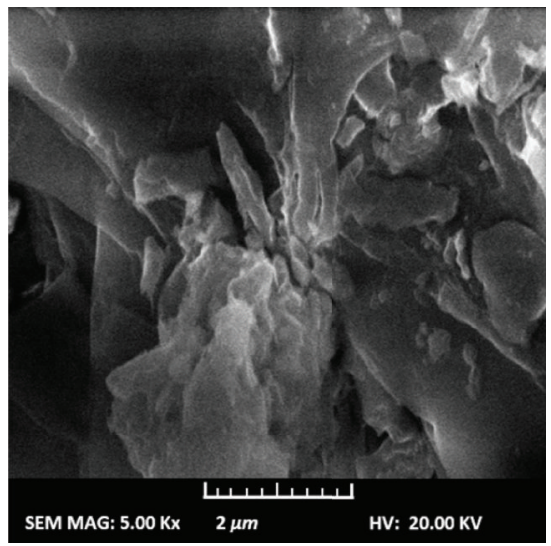

(b)

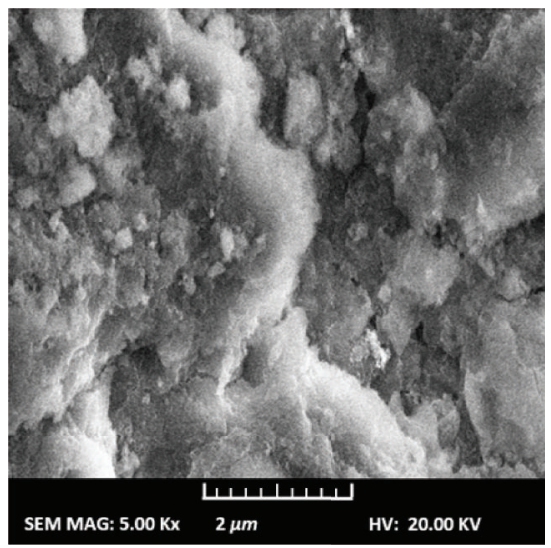

(c)

FIGURE 3: SEM diagrams: (a) CTS; (b) CTS-HMA; (c) ZvFe-CTSHMA.

Figure 3 compares the scanning electron micrographs of CTS, CTS-HMA, and ZvFe-CTS-HMA. The micrograph of chitosan indicates a smooth surface with some straps confirming high degree of chitin deacetylation as previously reported $[2,9]$. Chitosan loses its smooth surface on crosslinking with humic acid as indicated in the SEM images showing that intramolecular cross-linking between humic acid and chitosan was obtained and that secondary interactions 


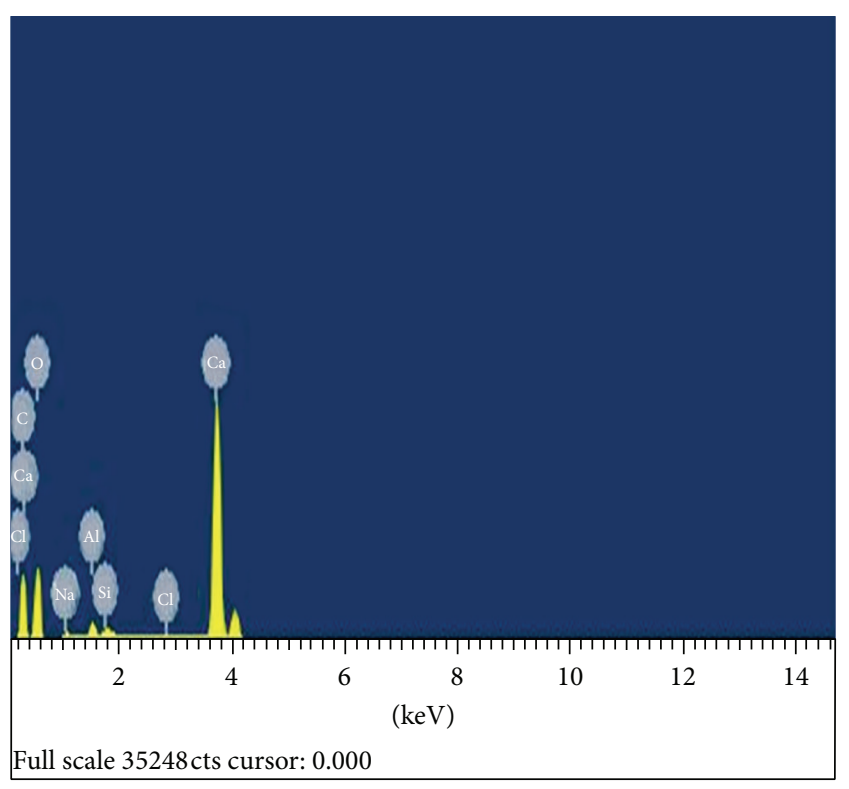

(a)

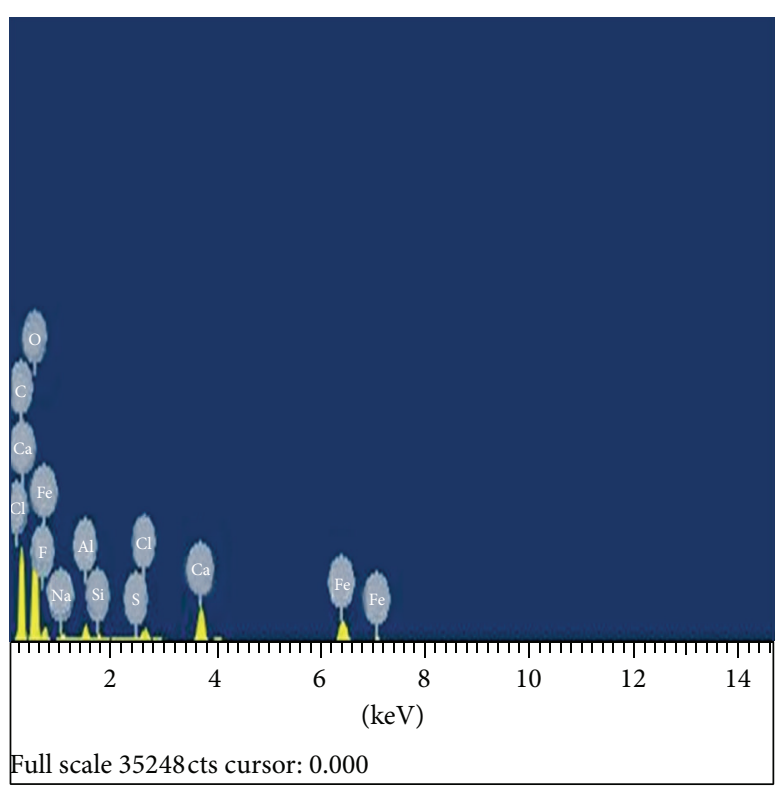

(b)

Figure 4: EDX spectra of (a) CTS-HMA (b) ZvFe-CTS-HMA.

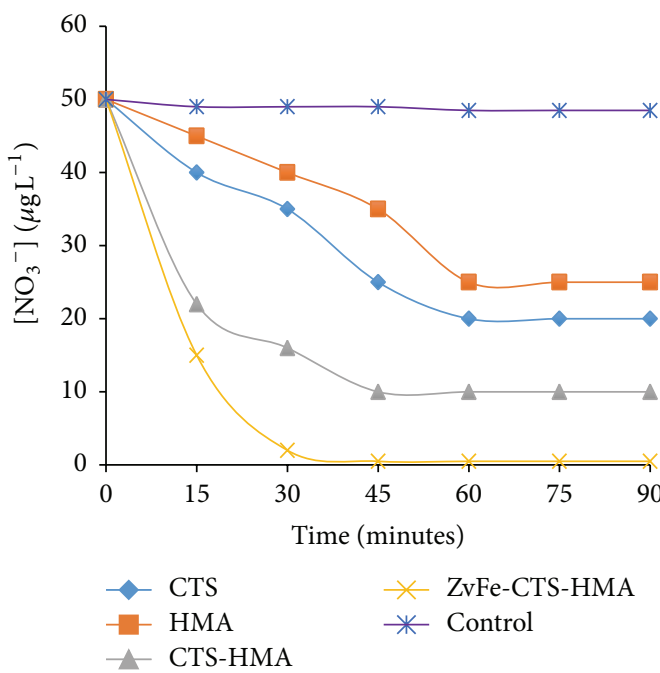

(a)

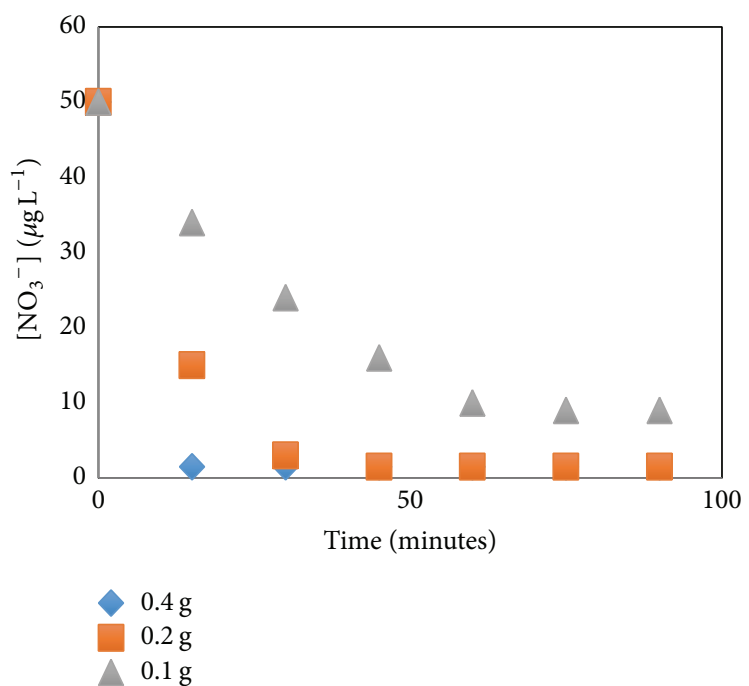

(b)

FIGURE 5: Reduction of $50 \mathrm{ppm}$ nitrate concentration using (a) $0.4 \mathrm{~g}$ dosages of control, CTS, HMA, CTS-HMA, and ZvFe-CTS-HMA. (b) $0.1 \mathrm{~g}, 0.2 \mathrm{~g}$, and $0.4 \mathrm{~g}$ dosages of ZvFe-CTS-HMA.

caused surface aggregation. This observation is in accord with our previous studies where chitosan has been crosslinked with notable cross-linkers [9]. The scanning image of ZvFe-CTS-HMA showed significant morphological changes thereby confirming a very strong bond between the crosslinked CTS-HMA and the zerovalent iron.

The characteristic peak of iron was observed in the EDX spectra ZvFe-CTS-HMA (Figure 4(b)) but was not seen in the EDX spectra of cross-linked CTS-HMA (Figure 4(a)). The electron dense part on surface condition of cross-linked chitosan was proved as iron. This method of elemental mapping of nanoparticle has been previously reported where it was used to confirm the existence of mercury ions before and after sorption on the electron dense part surface of aminated chitosan bead [21].

3.2. Reduction of Nitrate in Water. Batch experiment showed that rates of reduction of $\mathrm{NO}_{3}{ }^{-}$increased as the loadings of synthesized nanoparticle increased (Figure 5) and are attributed to the increase in the active sites on the chitosanzerovalent iron surface [22]. It was observed that the efficacy of humic acid to reduce nitrate showed a positive trend but at 


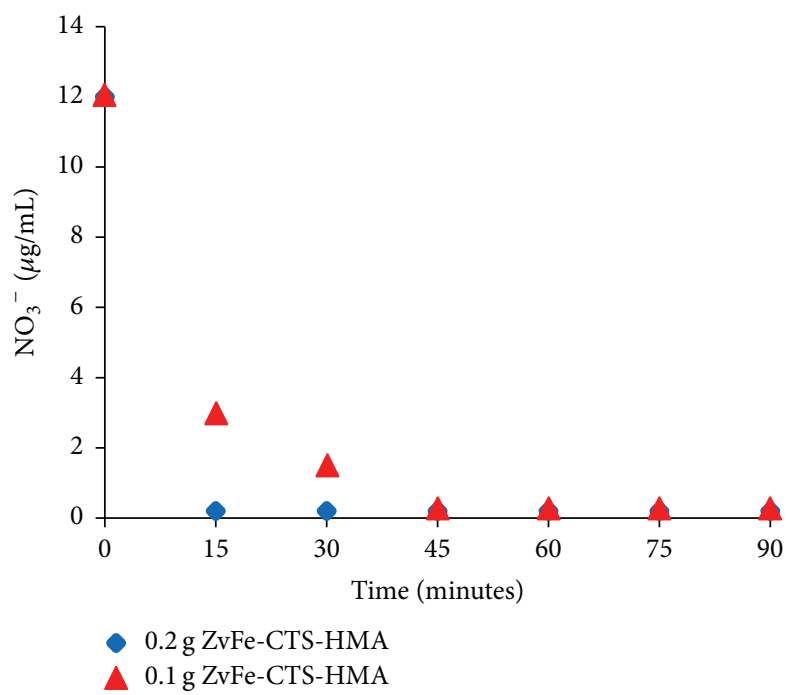

(a)

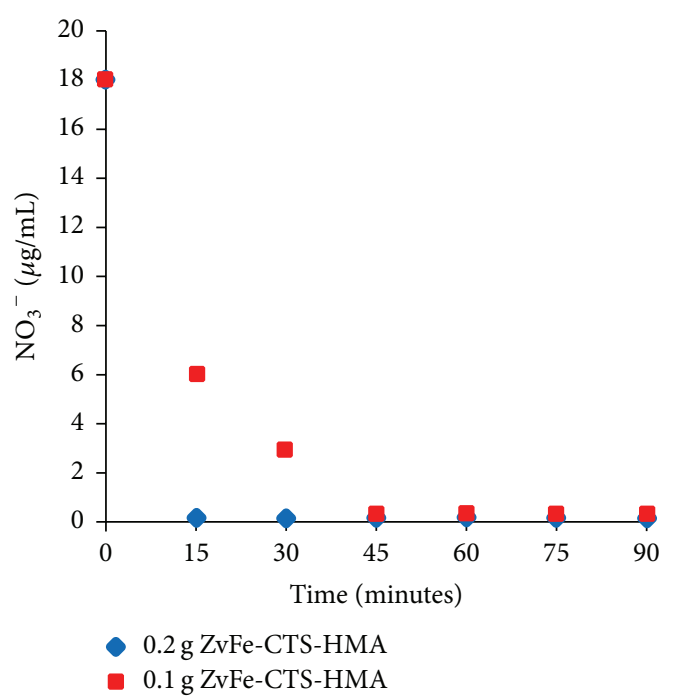

(b)

FIGURE 6: Nitrate reduction in (a) Ogun river water; (b) wastewater from drainages samples using $0.1 \mathrm{~g}$ and $0.2 \mathrm{~g}$ of zerovalent iron cross-linked chitosan nanoparticles.

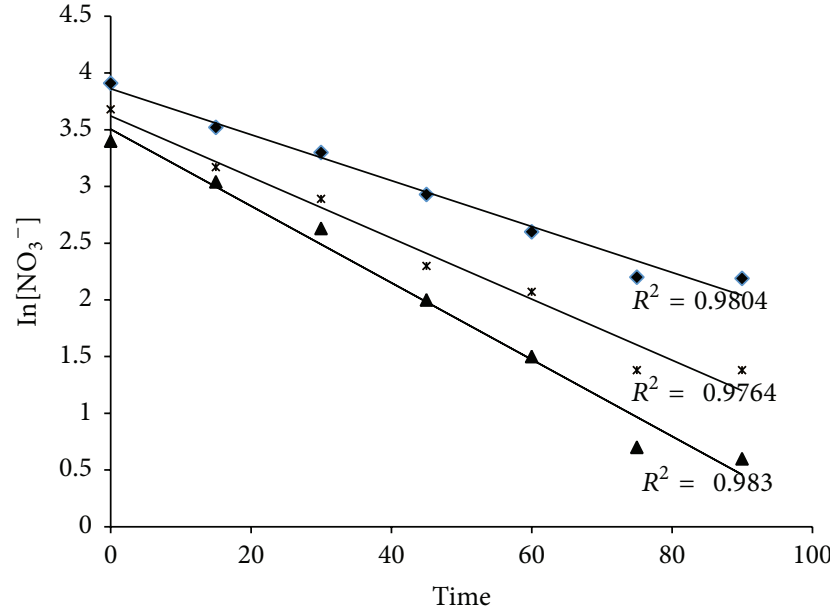

(a)

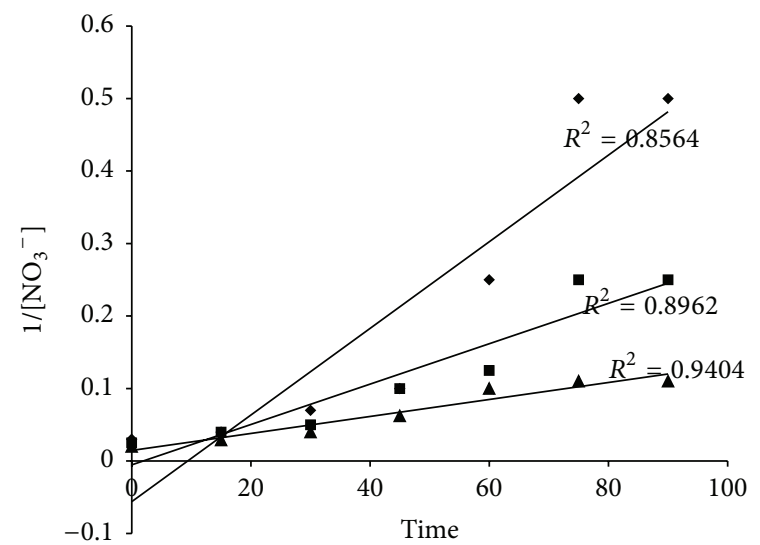

(b)

FIGURE 7: (a) Pseudofirst-order and (b) pseudosecond-order reaction plots for the reduction of nitrate using ZvFe-CTS-HMA.

a lower rate compared to chitosan, cross-linked chitosan, and zerovalent iron cross-linked chitosan (Figure 6). The zwitterionic character of humic acid allows the interaction of anions with its positively charged groups and cations with its negative charged groups [23]. This result confirmed the use of humic acid in fertilizer production to prevent nitrate leaching out into the groundwater [24].

3.3. Adsorption Isotherms. Equations (2) and (4) were performed for each data set of high nitrate concentration. The plots of the kinetic reaction models are displayed in Figure 7. The good fit of linear model was observed in the case of pseudofirst order with regression coefficient $\left(R^{2}\right)$ of 0.980 , 0.978 , and 0.983 , while the pseudosecond order gave $R^{2}$ value of $0.777,0.831$, and 0.896 . The observation implies that, under these conditions, rate reduction of nitrate is directly proportional to the concentration of the reactant; doubling the concentration increases the rate by a factor of 2 . This is comparable with some similar studies [25]. The Langmuir isotherm represents the experimental data better than the Freundlich isotherm (Figure 8). The regression coefficient of the Langmuir plot moving closer to unity provides strong evidence that the surface of ZvFe-CTS-HMA consists of adsorption sites. All adsorbed species interact only with the sites and not with each other with the adsorption being limited to a monolayer and independent of the presence of adsorbed species on the other sites.

Table 1 shows the model-fitted pseudofirst-order rate constants of nitrate reduction under various experimental conditions. For adsorption of the different $\mathrm{NO}_{3}{ }^{-}$concentration by ZvFe-CTS-HMA, $R_{L}$ values were calculated and the values are shown in Table 2 . In line with the isothermal shape 


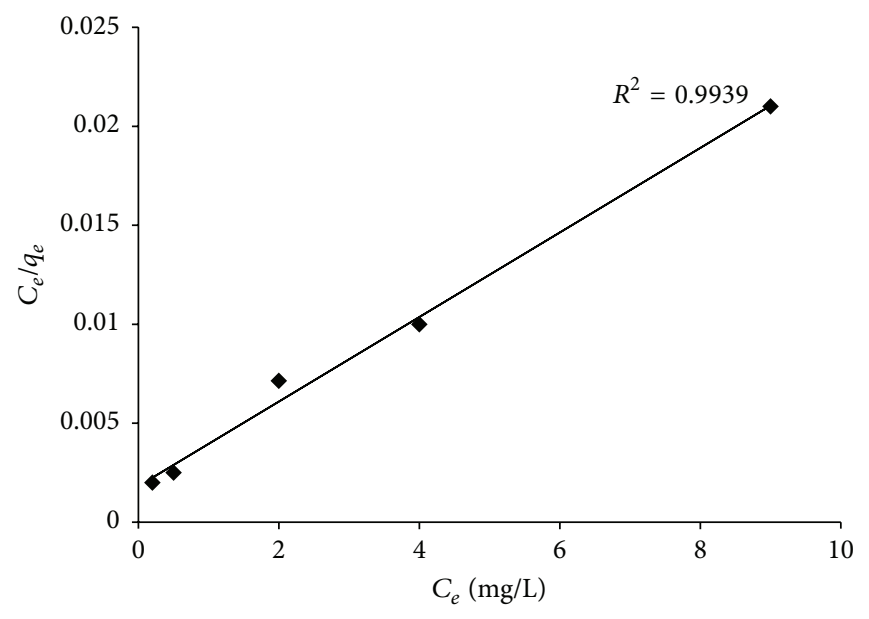

(a)

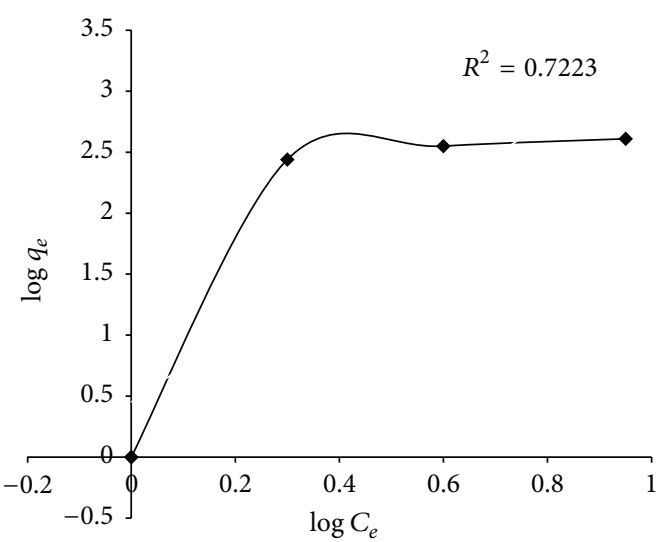

(b)

FIgURE 8: (a) Langmuir and (b) Freundlich plots for $\mathrm{NO}_{3}{ }^{-}$uptake using ZvFe-CTS-HMA.

TABLE 1: Model-fitted pseudofirst-order rate constants of nitrate reduction under various experimental conditions.

\begin{tabular}{ccccc}
\hline$\#$ & $\begin{array}{c}\text { ZvFe-CTS- } \\
\mathrm{HMA} / \mathrm{NO}_{3}{ }^{-}\end{array}$ & $\mathrm{NO}_{3}{ }^{-}$conc $(\mathrm{ppm})$ & $K_{\mathrm{obs}}\left(\mathrm{mins}^{-1}\right)$ & $R^{2}$ \\
\hline 1 & 0.0100 & 10 & 0.0399 & 0.6903 \\
2 & 0.0050 & 20 & 0.0424 & 0.8150 \\
3 & 0.0030 & 30 & 0.0335 & 0.9642 \\
4 & 0.0025 & 40 & 0.0269 & 0.9763 \\
5 & 0.0020 & 50 & 0.0207 & 0.9443 \\
6 & 0.0020 & 50 & 0.0207 & 0.9443 \\
7 & 0.0040 & 50 & 0.0377 & 0.7423 \\
8 & 0.0080 & 50 & 0.0250 & 0.3750 \\
9 & 0.0083 & 12 & 0.0469 & 0.8141 \\
10 & 0.0167 & 12 & 0.0292 & 0.3750 \\
11 & 0.0056 & 18 & 0.0417 & 0.8173 \\
12 & 0.0111 & 18 & 0.0321 & 0.3750 \\
13 & 0.0167 & 6 & 0.0287 & 0.5309 \\
14 & 0.0333 & 6 & 0.0243 & 0.3750 \\
\hline
\end{tabular}

obtained by $R_{L}$ values, a favorable adsorption of $\mathrm{NO}_{3}{ }^{-}$by $\mathrm{ZvFe}$-CTS-HMA took place (where all the calculated values fall within the favorable range of $0<R_{L}<1$ ) [17].

\section{Conclusions}

$\mathrm{ZvFe}$ nanoparticles were synthesized and dispersed on intramolecular cross-linked CTS-HMA stabilizer. The SEM micrograph of ZvFe-CTS-HMA nanocomposites distinctly showed interactions between the nanoparticles and the new amide chain of the chitosan-humic acid as well as the free amino groups on the chitosan outer surface. In line with previous studies, the use of cross-linked chitosan-humic acid as $\mathrm{ZvFe}$ stabilizers has greatly enhanced the reduction of nitrate ion reactivity. It was observed that reduction rate is directly
TABLE 2: $R_{L}$ values based on the Langmuir equation.

\begin{tabular}{lc}
\hline Nitrate initial & $R_{L}$ values \\
\hline Concentration $(\mathrm{ppm})$ & ZvFe-CTS-HMA \\
\hline 10 & 0.087 \\
20 & 0.045 \\
30 & 0.031 \\
40 & 0.023 \\
50 & 0.019 \\
\hline
\end{tabular}

proportional to the concentration of the reactant; doubling the concentration increases the rate by a factor of 2 . This nontoxic and biodegradable organic and inorganic polymeric composite can serve as a new material for permeable reactive barrier walls as well as a material for ex situ and in situ water treatment.

\section{Competing Interests}

The authors declare that they have no competing interests.

\section{Acknowledgments}

This work was supported by the Department of Chemistry, Federal University of Agriculture, Abeokuta, Nigeria.

\section{References}

[1] World Health Organization, Nitrate and Nitrite in Drinkingwater: Background Document for Development of WHO Guidelines for Drinking Water Quality, WHO Press, Geneva, Switzerland, 2011, http://www.who.int/water_sanitation_health/dwq/ chemicals/nitratenitrite2ndadd.pdf.

[2] S. Adewuyi, N. O. Sanyaolu, S. A. Amolegbe, A. O. Sobola, and O. M. Folarin, "Poly $[\beta$ - $(1 \rightarrow 4)$-2-amino-2-deoxy-D-ghicopyranose] based zero valent nickel nanocomposite for efficient reduction of nitrate in water," Journal of Environmental Sciences, vol. 24, no. 9, pp. 1702-1708, 2012. 
[3] H.-L. Lien, D. W. Elliott, Y.-P. Sun, and W.-X. Zhang, "Recent progress in zero-valent iron nanoparticles for groundwater remediation," Journal of Environmental Engineering and Management, vol. 16, pp. 371-380, 2006.

[4] L. Xie and C. Shang, "The effects of operational parameters and common anions on the reactivity of zero-valent iron in bromate reduction," Chemosphere, vol. 66, no. 9, pp. 1652-1659, 2007.

[5] Z. Xiong, D. Zhao, and G. Pan, "Rapid and controlled transformation of nitrate in water and brine by stabilized iron nanoparticles," Journal of Nanoparticle Research, vol. 11, no. 4, pp. 807-819, 2009.

[6] W.-H. Zhang, X. Quan, and Z.-Y. Zhang, "Catalytic reductive dechlorination of p-chlorophenol in water using $\mathrm{Ni} / \mathrm{Fe}$ nanoscale particles," Journal of Environmental Sciences, vol. 19, no. 3, pp. 362-366, 2007.

[7] M. Kumar and S. Chakraborty, "Chemical denitrification of water by zero-valent magnesium powder," Journal of Hazardous Materials, vol. 135, no. 1-3, pp. 112-121, 2006.

[8] F. He and D. Y. Zhao, "Preparation and characterization of a new class of starch-stabilized bimetallic nanoparticles for degradation of chlorinated hydrocarbons in water," Environmental Science and Technology, vol. 39, no. 9, pp. 3314-3320, 2005.

[9] C. A. Akinremi, V. B. Oyelude, S. Adewuyi, S. A. Amolegbe, and T. Arowolo, "Reduction of bromate in water using zerovalent cobalt 2,6-pyridine dicarboxylic acid crosslinked chitosan nanocomposite," Journal of Macromolecular Science Part A, vol. 50, no. 4, pp. 435-440, 2013.

[10] M. V. Yudov, D. M. Zhilin, A. P. Pankova et al., "Synthesis, metal-binding properties and detoxifying ability of sulphonated humic acids," in Use of Humic Substances to Remediate Polluted Environments: From Theory to Practice, I. V. Perminova, K. Hatfield, and N. Hertkorn, Eds., pp. 485-498, Springer, Amsterdam, The Netherlands, 2005.

[11] S. Erdogan, A. Baysal, O. Akba, and C. Hamamci, "Interaction of metals with humic acid isolated from oxidized coal," Polish Journal of Environmental Studies, vol. 16, no. 5, pp. 671-675, 2007.

[12] A. K. Pandey, S. D. Pandey, and V. Misra, "Stability constants of metal-humic acid complexes and its role in environmental detoxification," Ecotoxicology and Environmental Safety: Environmental Research, Section B, vol. 47, no. 2, pp. 195-200, 2000.

[13] X. Zhang and R. Bai, "Mechanisms and kinetics of humic acid adsorption onto chitosan-coated granules," Journal of Colloid and Interface Science, vol. 264, no. 1, pp. 30-38, 2003.

[14] A. Domard and M. Rinaudo, "Preparation and characterization of fully deacetylated chitosan," International Journal of Biological Macromolecules, vol. 5, no. 1, pp. 49-52, 1983.

[15] M. Bodnar, J. F. Hartmann, and J. Borbely, "Synthesis and study of cross-linked chitosan-N-poly(ethylene glycol) nanoparticles," Biomacromolecules, vol. 7, no. 11, pp. 3030-3036, 2006.

[16] APHA, Standard Methods for the Examination of Water and Wastewater, American Public Health Association, 1999.

[17] W. S. Wan Ngah, C. S. Endud, and R. Mayanar, "Removal of copper(II) ions from aqueous solution onto chitosan and crosslinked chitosan beads," Reactive and Functional Polymers, vol. 50, no. 2, pp. 181-190, 2002.

[18] U.-T. Santosa, S. J. Santosa, D. Siswanta, B. Rusdiarso, and S. Shimazu, "Characterization of sorbent produced through immobilization of humic acid on chitosan using glutaraldehyde as cross-linking agent and $\mathrm{Pb}(\mathrm{II})$ ion as active site protector," Indonesian Journal of Chemistry, vol. 10, no. 3, pp. 301-309, 2010.
[19] C.-Y. Chen, T.-H. Chang, J.-T. Kuo, Y.-F. Chen, and Y.-C. Chung, "Characteristics of molybdate-impregnated chitosan beads (MICB) in terms of arsenic removal from water and the application of a MICB-packed column to remove arsenic from wastewater," Bioresource Technology, vol. 99, no. 16, pp. 74877494, 2008.

[20] Y.-P. Sun, X.-Q. Li, J. Cao, W.-X. Zhang, and H. P. Wang, "Characterization of zero-valent iron nanoparticles," Advances in Colloid and Interface Science, vol. 120, no. 1-3, pp. 47-56, 2006.

[21] C. Jeon and W. H. Höll, "Chemical modification of chitosan and equilibrium study for mercury ion removal," Water Research, vol. 37, no. 19, pp. 4770-4780, 2003.

[22] L. Chen, C.-Y. Tang, N.-Y. Ning, C.-Y. Wang, Q. Fu, and Q. Zhang, "Preparation and properties of chitosan/lignin composite films," Chinese Journal of Polymer Science, vol. 27, no. 5, pp. 739-746, 2009.

[23] E. M. Peña-Méndez, J. Havel, and J. Patočka, "Humic substances compounds of still unknown structure: applications in agriculture, industry, environment, and biomedicine," Journal of Applied Biomedicine, vol. 3, no. 13, article 24, 2005.

[24] F.-C. Liu, S.-J. Xing, C.-H. Duan, Z.-Y. Du, H.-L. Ma, and B.Y. Ma, "Nitrate nitrogen leaching and residue of humic acid fertilizer in field soil," Huanjing Kexue, vol. 31, no. 7, pp. 1619$1624,2010$.

[25] H. Park, Y.-M. Park, K.-M. Yoo, and S.-H. Lee, "Reduction of nitrate by resin-supported nanoscale zero-valent iron," Water Science and Technology, vol. 59, no. 11, pp. 2153-2157, 2009. 

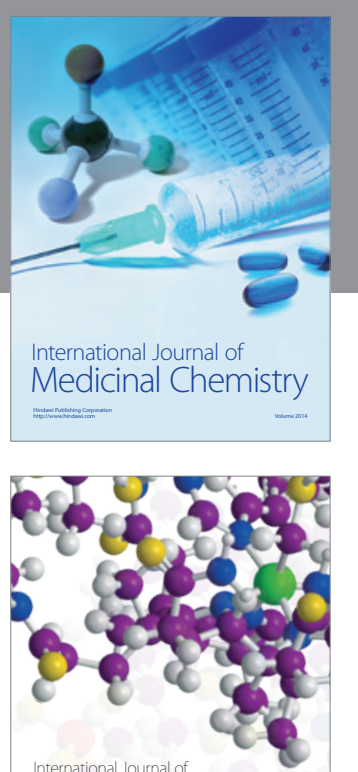

Carbohydrate Chemistry

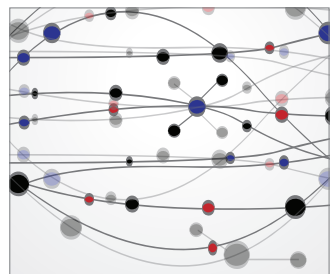

The Scientific World Journal
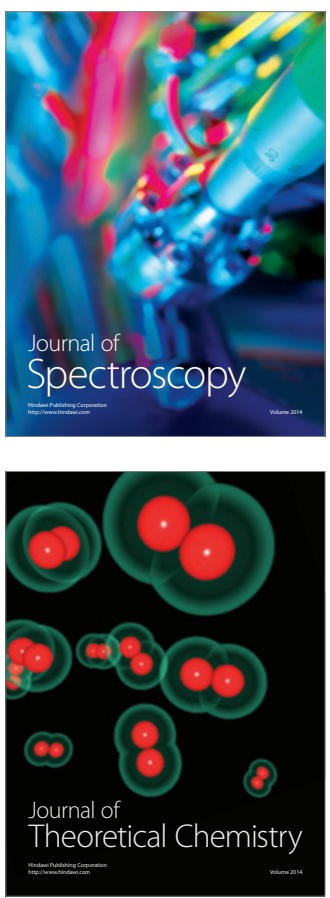
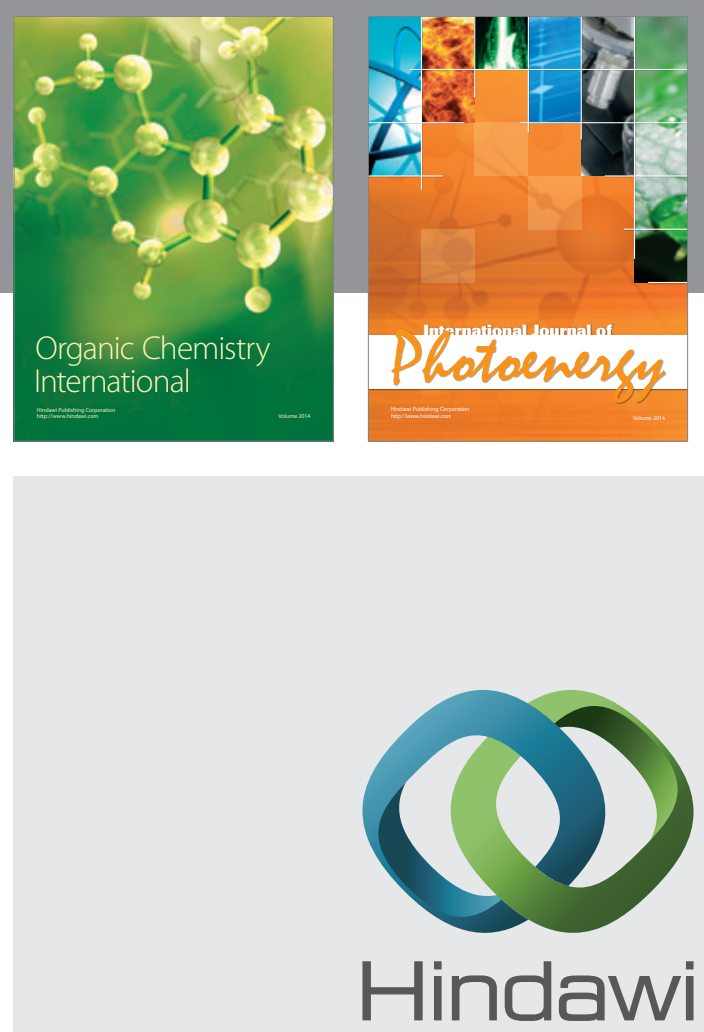

Submit your manuscripts at

http://www.hindawi.com

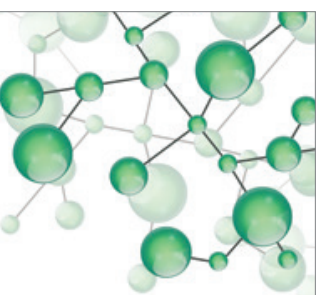

International Journal of

Inorganic Chemistry

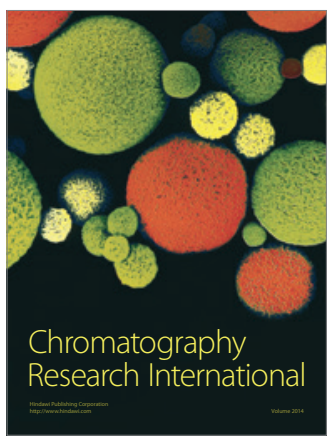

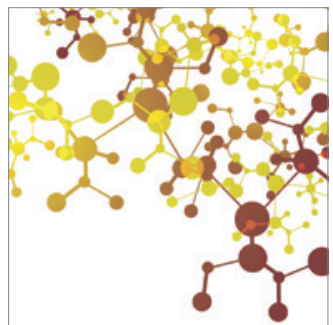

Applied Chemistry
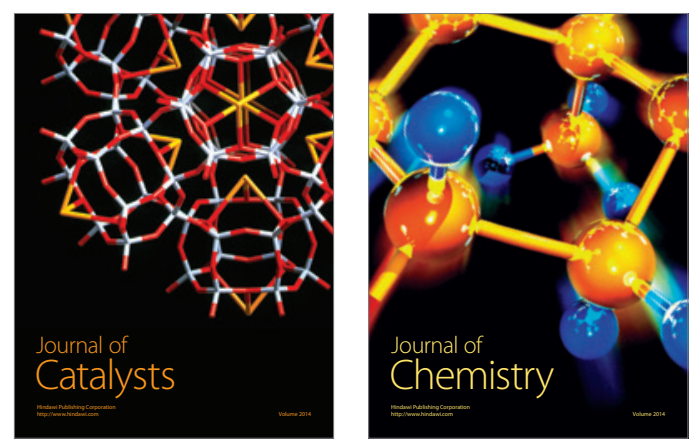
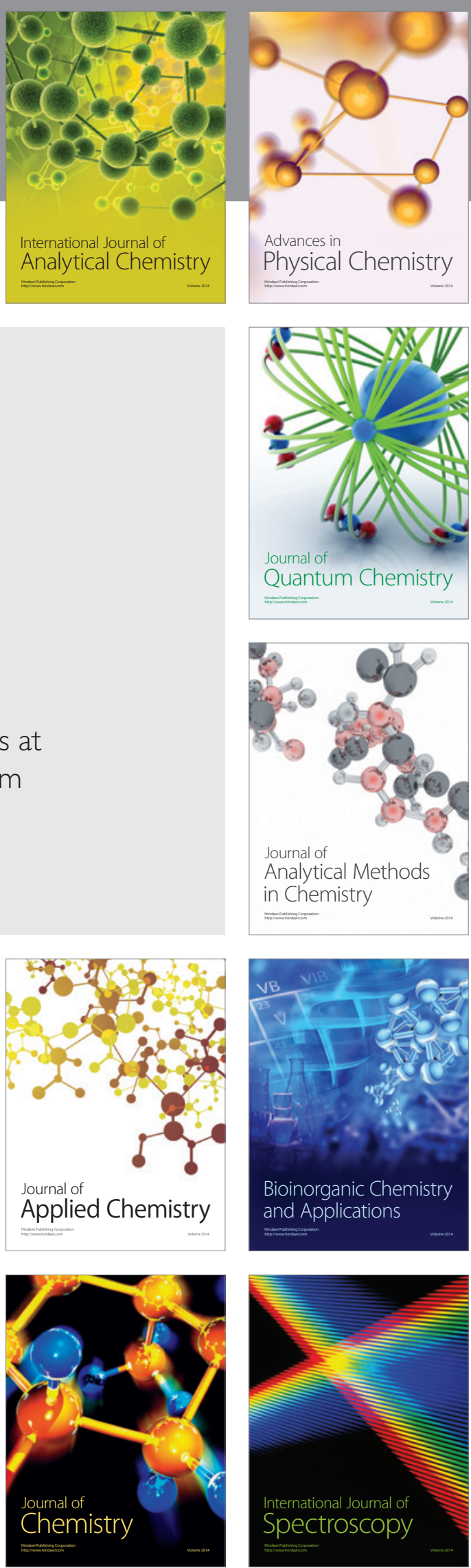